\title{
Australian Early Psychosis Research Network: national collaboration, international competitive advantage
}

\section{An expansion of clinical infrastructure for early psychosis intervention offers new possibilities for research, treatment and service development}

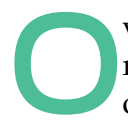

ver the past three decades, Australian

researchers have pioneered a conceptual model

of the clinical stages underpinning progression of potentially serious mental disorders, including psychosis. This approach, supported by growing evidence, has informed the development of new service models focusing on the mental health needs of young people.

By shifting the focus of the health sector to intervention during the early and less specific stages of mental disorders, substantial conceptual and practical progress has been made. ${ }^{1}$ This progress has generated optimism, buttressed by growing evidence of the effectiveness and cost-effectiveness of early intervention in psychosis from clinical trials and health services research conducted in Australia and around the world. ${ }^{2-4}$

\section{"The ultimate goal ... is substantial improvement in health and social outcomes for people living with psychosis"}

Early intervention has demonstrated potential to reduce the effects of illness on age-specific developmental and occupational goals. It can also improve social and economic participation and productivity. Programs such as headspace have been scaled up across Australia, and have already improved access and engagement in stigma-free, youth-friendly settings. ${ }^{5}$ The federal government's planned expansion of comprehensive early psychosis programs in every state and territory ${ }^{6}$ offers an unprecedented opportunity to promote innovative large-scale, multicentre research into the early stages of psychotic illness.

Against the backdrop of this expansion of innovative service delivery models, we face ongoing challenges. We have an incomplete understanding of what causes psychosis. Even when the best available treatments are delivered early and with precision, longer-term clinical and functional outcomes remain suboptimal. People with long-term psychotic illness die up to 20 years earlier than others. ${ }^{7}$ Our patients and their families expect better results.
Patrick D McGorry MD, PhD, FRANZCP ORYGEN Youth Health Research Centre, Melbourne, VIC.

pmcgorry@ unimelb.edu.au On behalf of the Australian Early Psychosis Research Network Writing Group doi: 10.5694/mjal4.00914

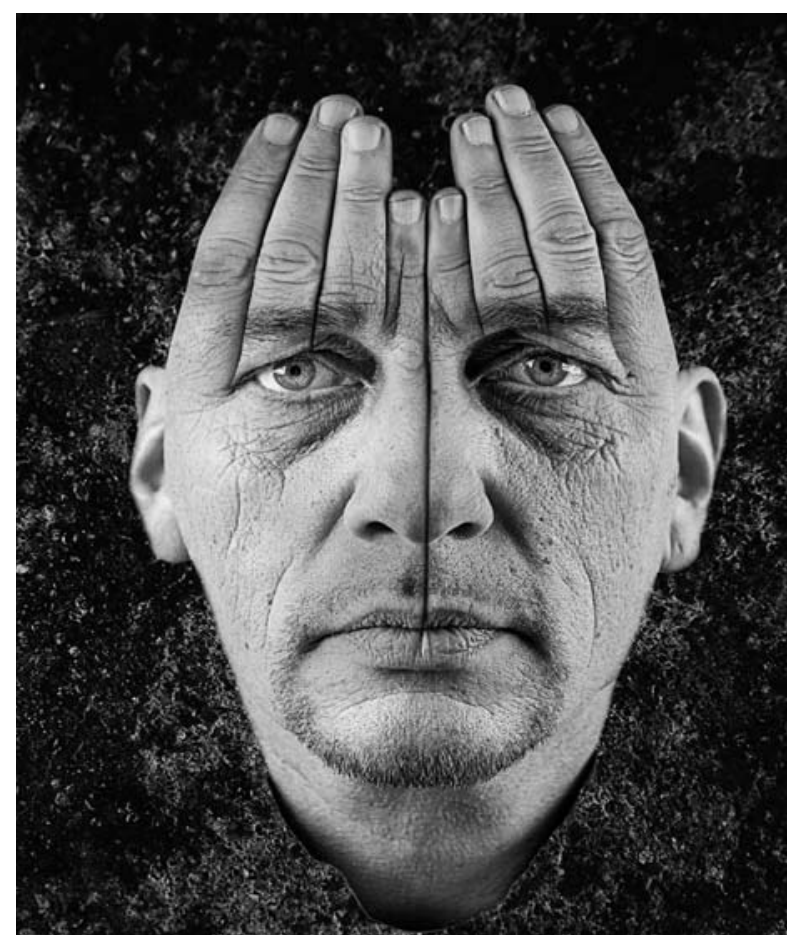

However, the situation we face is not too different from that seen in oncology in previous years, when many standard treatments were associated with substantial toxicity, delivery systems were variable and most patients presented in the later stages of illness. Yet, breakthroughs aside, strategies such as early detection, timely intervention and sustained delivery of highquality care throughout "critical" illness periods can substantially improve outcomes.

Stimulated by the federal government's investment in comprehensive clinical infrastructure for early psychosis intervention, a large group of Australian psychosis researchers formed the Australian Early Psychosis Research Network (Appendix). At a recent meeting of this group, research priorities were discussed.

The group agreed that there is an urgent need for novel treatment strategies that are safer, more effective and more acceptable to patients and their families. Current candidates include omega-3 fatty acids, aspirin, $\mathrm{N}$-acetylcysteine, sodium benzoate, cannabidiol and oestrogen. In the early stages of illness, there is a greater degree of clinical equipoise: the risk-benefit ratio can be unclear, with more evidence required on the effectiveness and safety of treatments. This 
situation creates momentum for a sequence of stepwise clinical trials, as follows:

- The study of biomarkers to guide the use of such therapies using a strategy in which "target engagement" is assessed as a predictor of treatment response. This means demonstrating not only whether novel therapies work, but also how they work. This approach directly tackles the problem of heterogeneity within clinical samples assembled using current syndromal diagnostic criteria. Personalised medicine is the ultimate goal.

- The further development of novel psychosocial interventions, including online platforms, which focus predominantly on functional recovery. These are particularly promising in terms of consumer engagement, clinical effectiveness and cost-effectiveness. Their integration with biological therapies and linkage with stages of illness require exploration.

- The examination of how to reduce the elevated risks of premature mortality (from both cardiovascular disease and suicide) from the time of onset of psychotic illness.

There is also a need for health services research to determine not only which interventions are effective and cost-effective, but in what context. Treatment fidelity in the real world has not been studied well, and the range and quality of clinical interventions vary between and within services. Studying the effect of culture of care and model fidelity is also critical.

The ultimate goal of the Australian Early Psychosis Research Network is substantial improvement in health and social outcomes for people living with psychosis. We have an opportunity to develop a new culture in mental health that has the capacity for largescale clinical trials with embedded biomarker research, on a par with what exists elsewhere in medicine. Federal government reform and investment gives Australia a unique competitive advantage in this. We do not intend to let this opportunity pass.

Competing interests: No relevant disclosures.

Provenance: Not commissioned; externally peer reviewed. 
1 McGorry PD. The next stage for diagnosis: validity through utility. World Psychiatry 2013; 12: 213-215.

2 Bertelsen M, Jeppesen P, Petersen L, et al. Five-year followup of a randomized multicenter trial of intensive early intervention vs standard treatment for patients with a first episode of psychotic illness: the OPUS trial. Arch Gen Psychiatry 2008; 65: 762-771.

3 Hegelstad WT, Larsen TK, Auestad B, et al. Long-term followup of the TIPS early detection in psychosis study: effects on 10-year outcome. Am J Psychiatry 2012; 169: 374-380.

4 Henry LP, Amminger GP, Harris MG, et al. The EPPIC followup study of first-episode psychosis: longer-term clinical and functional outcome 7 years after index admission. J Clin Psychiatry 2010; 71: 716-728.

5 Rickwood DJ, Telford NR, Parker AG, et al. headspace - Australia's innovation in youth mental health: who are the clients and why are they presenting? Med J Aust 2014; 200: 108-111.

6 Hughes F, Stavely H, Simpson R, et al. At the heart of an early psychosis centre: the core components of the 2014 Early Psychosis Prevention and Intervention Centre model for Australian communities. Australas Psychiatry 2014; 22: 228-234.

7 Thornicroft G. Physical health disparities and mental illness: the scandal of premature mortality. Br J Psychiatry 2011; 199: 441-442. 